\title{
Las bajas dosis de estrógenos conjugados con o sin progesterona son efectivas para mantener la masa ósea en la postmenopausia reciente
}

Effect of Lower Doses of Conjugated Equine Estrogens With and Without Medroxyprogesterone Acetate on Bone in Early Postmenopausal Women. Lindsay R, Gallagher C, Kleerekoper M. JAMA 2002; 287: 2668-2676.

\begin{abstract}
Objetivo
Evaluar el efecto de bajas dosis de estrógenos conjugados equinos (CEE) solos o combinados con acetato de medroxiprogesterona (MPA) sobre la densidad mineral ósea (DMO) de columna y fémur, sobre el contenido mineral de cuerpo entero (BMC) y sobre los marcadores del-recambio óseo en la mujer menopáusica.
\end{abstract}

\section{Diseño}

Estudio clínico randomizado, doble ciego de 2 años de seguimiento.

\section{Lugar}

Estudio realizado en 19 centros de EE.UU. entre 1995-2000.

\section{Pacientes}

Participaron 822 mujeres postmenopáusicas (por lo menos un año de amenorrea) entre 40-65 años, con $\mathrm{FSH}>30 \mathrm{mUI} / \mathrm{mL}$ y estradiol $<50 \mathrm{pg} / \mathrm{mL}$, si poseían útero y dentro del $20 \%$ del peso ideal.

\section{Intervención}

Las pacientes se randomizaron a recibir uno de 8 tratamientos: 102 pacientes CEE $0.625 \mathrm{mg} / \mathrm{d}$; 92 pacientes CEE + MPA $(0.625 \mathrm{mg} / \mathrm{d}$ + $2.5 \mathrm{mg} / \mathrm{d}$ ); 101 pacientes CEE $0.450 \mathrm{mg} / \mathrm{d} ; 104$ pacientes CEE + MPA ( $0.450 \mathrm{mg} / \mathrm{d}+2.5 \mathrm{mg} / \mathrm{d}) ; 98$ pacientes CEE + MPA ( 0.450 $\mathrm{mg} / \mathrm{d}+1.5 \mathrm{mg} / \mathrm{d}) ; 94$ pacientes CEE $0.3 \mathrm{mg} / \mathrm{d} ; 105$ pacientes CEE + MPA ( $0.3 \mathrm{mg} / \mathrm{d}+1.5 \mathrm{mg} / \mathrm{d})$ y 101 pacientes con placebo. Todas recibieron suplemento de $600 \mathrm{mg} / \mathrm{día}$ de carbonato de calcio.

\section{Medición de los resultados principales}

Densidad mineral ósea (raquis, fémur total y BMC medidos cada 6 meses) y marcadores bioquímicos (osteocalcina sérica como marcador de formación y $\mathrm{N}$-Telopéptidos urinarios, indicador de resorción) dosados cada semestre.

\section{Resultados principales}

En todos los grupos de tratamiento activo, luego de 24 meses se observaron incrementos de la DMO, expresado como porcentaje de aumento a partir del basal. Mientras que en el grupo placebo se observó perdida de masa ósea.

En columna lumbar: CEE $0.625 \mathrm{mg} / \mathrm{d}$ vs $0.3 \mathrm{mg} / \mathrm{d}$ presentó diferencias significativas $(2.43 \%$ vs $1.33 \% p=0.02)$. El agregado de MPA $2.5 \mathrm{mg} / \mathrm{d}$ determinó mayor ganancia ósea en los grupos de CEE de dosis más altas. Para CEE $0.625 \mathrm{mg} / \mathrm{d}+$ MPA $2.5 \mathrm{mg} / \mathrm{d}$ fue $3.46 \%$ vs $2.43 \%$ para CEE solo $(p=0.03)$ y para CEE $0.45 \mathrm{mg} / \mathrm{d}$ + MPA $2.5 \mathrm{mg} 7 \mathrm{~d}$ fue $3.01 \%$ vs $2.09 \%$ para CEE solo $(p=0.05)$. No existió diferencia con el agregado de MPA $1.5 \mathrm{mg} / \mathrm{d}$.

En cadera total hubo significativo incremento de la DMO a partir del basal en todos los grupos de tratamiento $(p=<0.05)$. No se verificó diferencia entre los grupos de CEE solos o asociados a MPA en dosis comparables. En cuerpo entero también se registró incremento en todos los grupos de tratamientos $(p<0.001)$ excepto para $0.3 \mathrm{mg} / \mathrm{d}$ de CEE. Tampoco hubo diferencia con el agregado de MPA.

MARCADORES BIOQUÍMICOS: N-Telopéptido y Osteocalcina descendieron su concentración en todos los grupos tratados $(p<0.001)$ respecto del basal. El porcentaje de reducción estuvo directamente relacionado con la dosis recibida. El descenso fue más acentuado en los 6 primeros meses. El grupo placebo no mostró cambios en los marcadores luego de 2 años de seguimiento.

Los efectos adversos fueron elevados en ambos grupos, más marcados en el activo ( $96 \%$ de los pacientes). Hiperplasia endometrial, metrorragias y sangrado vaginal fueron los más frecuentes $(p<0.05)$ en el grupo de CEE solo y estuvo directamente relacionado con la dosis $(p<0.05)$. Mastalgia y sangrado vaginal fueron referidos en el grupo de CEE-MPA ( $p<0.05)$ vs placebo. Los efectos adversos determinaron la suspensión del tratamiento en $9 \%$ del placebo y $16 \%$ del grupo activo.

\section{Conclusiones}

Este es el primer estudio randomizado, controlado para evaluar el efecto de bajas dosis de CEE solos o combinados con MPA sobre la DMO. Los resultados muestran prevención de la pérdida de masa ósea y reducción de los marcadores de recambio óseo en mujeres de menopausia reciente. Resultados similares se encontraron en mujeres añosas. Sólo en raquis se verificó una respuesta en relación directa con las dosis usadas y con la asociación con MPA, explicable por la mayor actividad metabólica del hueso trabecular. La falta de modificaciones en los marcadores bioquímicos del grupo placebo manifiesta que la dosis de calcio era óptima.

Fuente de financiamiento: Wyeth Research.

\section{Comentario}

Este estudio muestra que en pacientes menopáusicas recientes las dosis bajas de estrógenos, solos o combinados con dosis bajas de MPA, pueden mantener y elevar la masa ósea con pocos efectos adversos. Cabe destacar que en el presente estudio prácticamente todas las pacientes tenían DMO normal y que los valores estrogénicos basales no eran tan bajos.

En mujeres añosas con TRH a bajas dosis y con buen aporte de calcio y vitamina $\mathrm{D}$, también existe mejoría de DMO ${ }^{1}$.

Estudios recientes en menopáusicas demostraron que bajas concentraciones de estrógenos endógenos $(10-25 \mathrm{pg} / \mathrm{ml})$ se acompañan de mayor DMO y menor riesgo de fracturas que cuando las concentraciones son indetectables $(<5 \mathrm{pg} / \mathrm{ml})^{2}$.

Probablemente THR a dosis bajas con menores efectos adver- $\operatorname{sos}^{3}$, produzca mayor adaptación al tratamiento, fundamentalmente en la actualidad donde los resultados de la combinación CEE + MPA ( $0.625 \mathrm{mg} / \mathrm{d}+2.5 \mathrm{mg} / \mathrm{d}$ respectivamente) han mostrado mayor incidencia de efectos indeseables (carcinoma mamario y eventos cardiovasculares) ${ }^{4}$

Deberá establecerse si estas dosis son efectivas para pacientes con osteoporosis densitométrica por la falta de estudios a largo plazo y porque aún no se ha demostrado que disminuyan la incidencia de fracturas, requisito necesario para aceptar una droga para el tratamiento de la osteoporosis. Si es dosis efectiva ¿cuánto tiempo de tratamiento para mantener el efecto logrado?, son interrogantes todavía no aclarados. Si está claro que siempre debe ser acompañada de buen aporte de calcio (alimentario y/o medicamentoso) y vitamina $\mathrm{D}$.

\section{Dra. Ana María Galich [ Servicio de Endocrinología, Metabolismo y Medicina Nuclear. Hospital Italiano de Buenos Aires ]}

\section{Referencias}

1. The Effect of Low-Dose Continuous Estrogen and Progesterone Therapy with Calcium and Vitamin D on Bone in Elderly Women. Recker RR, Davies KM, Dowd RM, Heaney RP. Ann Intern Med 1999; 130: 897-904.

2. Associations between Low Levels of Serum Estradiol, Bone Density, and Fractures among Elderly Women: The Study of Osteoporotic Fractures. Ettinger B, Pressman A, Sklarin P y col. J Clin Endocrinol Metab 1998; 83: 2239-2243.

3. Low-Dose Esterified Estrogen Therapy. Effects on Bone, Plasma Estradiol Concentrations, Endometrium and Lipid Levels. Genant HK, Lucas J, Weiss S y colab. Arch Intern Med 1997; 157: 26092615 .

2615. Health Initiative Investigators. JAMA 2002; 288: 321-333. 